\title{
Literature Reviews
}

\section{Systematic Review of the Proposed Associations between Physical Exercise and Creative Thinking}

\author{
Emily Fritha, Seungho Ryu ${ }^{\mathrm{b}}$, Minsoo Kang ${ }^{\mathrm{b}}$, Paul D. Loprinzi ${ }^{*_{a}}$
}

[a] Exercise \& Memory Laboratory, Department of Health, Exercise Science and Recreation Management, The University of Mississippi, University, MS, USA. [b] Health and Sports Analytics Laboratory, Department of Health, Exercise Science and Recreation Management, The University of Mississippi, University, MS, USA.

\begin{abstract}
The objective of this study was to evaluate the association between physical exercise and creative thinking. A systematic review approach was employed by searching PubMed, Google Scholar and Psychlnfo databases. Among the evaluated 13 studies, $92 \%$ indicated a beneficial relationship. However, $77 \%$ were vulnerable to moderate-high risk for methodological bias, suggesting adherence to standardized and controlled research initiatives should be promoted. There appears to be weak to modest support for acute, moderateintensity exercise to benefit creativity. Exercise timing relative to creativity assessment protocols should be addressed and further detailed. Creativity scoring procedures must be refined, and an increased focus on the motivational components of exercise may help guide researchers in measuring creative thoughts and behavior. Broader concluding claims that creativity, in general, is improved or impaired by exercise, is as problematic as sweeping statements that exercise improves or impairs a measure as dynamic as intelligence. Scientific inquiries must specify precisely which outcome characteristics are changing in line with research interventions. This review identifies several fallible linkages between physical activity and creativity. Too few studies were conducted on strong methodological foundations, perpetuating the risk for undermining or inaccurately inflating the potential association between exercise and creative thinking behavior.
\end{abstract}

Keywords: cognition, exercise psychology, health promotion, innovation, mental health, physical activity, research methods

Europe's Journal of Psychology, 2019, Vol. 15(4), 858-877, https://doi.org/10.5964/ejop.v15i4.1773

Received: 2018-09-08. Accepted: 2019-03-24. Published (VoR): 2019-12-19.

Handling Editor: Natalia Wentink Martin, University of Liverpool, UK

${ }^{*}$ Corresponding author at: Exercise \& Memory Laboratory, Department of Health, Exercise Science, and Recreation Management, School of Applied Sciences, The University of Mississippi, 229 Turner Center, University, MS 38677, USA. Tel: +662 915 5521. E-mail: pdloprin@olemiss.edu (https://creativecommons.org/licenses/by/4.0/), which permits unrestricted use, distribution, and reproduction in any medium, provided the original work is properly cited.

Scientific inquiry in any field is difficult when the parameter beneath the lens of empirical scrutiny is difficult to both operationalize and localize. Researchers have attempted to define creativity as a broad construct that encompasses "the degree of novelty of which the person is capable, or which he [or she] habitually exhibits" (Guilford, 1950). More recently, the intricate processes of creativity have been posited to correspond with "our ability to change existing patterns, break with the present, and build something new" (Dietrich \& Kanso, 2010). The variegated outcomes of such creative processes have also been described as products which "can be tested in terms of the frequency of uncommon, yet acceptable, responses to items" (Guilford, 1950). Further, a theme of societal relevance, or value, is proposed as a crucial standard for creative production, as "the creative work is a novel work that is accepted as tenable or useful or satisfying by a group in some point in time" (Stein, 1953). 
Despite the volume and remarkable adaptability of creative exposition, Guilford revolutionized empirical creativity assessment with a push to evaluate creative divergent thinking (Guilford, 1950). The examination of divergent thinking, or the creative act of generating multiple solutions from a single stimulus (Berkowitz, 2014), continued to serve as a pervasive staple for the best measurement practices in creativity studies for decades, and is still widely investigated in modern research. Indeed, it is well established that divergent thinking is a tool for predicting creative potential (Runco, 2008). However, it is certainly not the only tool that should be wielded by scientists searching for causal relationships. For example, convergent thinking, or solving a task with one correct solution, is also suggested to play a large role in explaining the nature of creative thought (Berkowitz, 2014). By the late 1990's, creativity research diverted from a narrowed focus on the evaluation of divergent thinking, and began to encompass a broader range of scientific analysis, including neuroscientific correlates, personality, insight, and other systems-based approaches exposing important creativity measurement outcomes (Csikszentmihalyi, 1999; Mumford, 2003).

Unfortunately, as creativity research efforts in psychological and neurobiological disciplines appear to be making headway towards the practical conceptualization of such an untenable construct, creativity research in exercise science and health promotion is stunted. The lack of experimental work on this topic is staggering, with only 13 research studies investigating the associations between physical exercise and quantifiable creative products (Blanchette, Ramocki, O'del, \& Casey, 2005; Colzato et al., 2013; Curnow \& Turner, 1992; Gondola, 1986, 1987; Gondola \& Tuckman, 1985; Herman-Tofler \& Tuckman, 1998; Hinkle, Tuckman, \& Sampson, 1993; Oppezzo \& Schwartz, 2014; Ramocki, 2002; Steinberg et al., 1997; Tuckman \& Hinkle, 1986; Zhou, Zhang, Hommel, \& Zhang, 2017). Over half of this body of literature was published prior to the millennium, and, as demonstrated herein, the vast majority lack sound rationale and methodological quality.

Following the systematic review framework detailed elsewhere (Murad et al., 2014), this systematic review will provide a detailed synthesis of the exercise and creativity work accomplished thus far. The dearth of unbiased research on exercise and creativity is a critical issue, which must be prevented for future development in this area to continue unencumbered by obstruction, or even absence, of meaningful evidence to answer the pervasive question, "Does exercise influence creative potential?" Therefore, a secondary aim of this review is to direct future experimentation towards more informed, and applicable, methods of inquiry, and provide direction for identifying prudent questions worth answering in the field.

\section{Method}

\section{Inclusion Criteria}

Research studies were included if they utilized an experimental study design, were published in English, indexed in PubMed, Google Scholar and Psyclnfo, and specifically evaluated the influence of acute or habitual physical exercise on creativity in children or adults, of either gender and with no known psychological or physical limitations or preexisting pathology that would prevent them from being classified as healthy at baseline. Any exercise intervention (acute or chronic laboratory or free-living physical activities) coupled with either an active or traditional control group (no exercise) was considered. 


\section{Outcome measure}

Cognitive creativity (analogy, convergent thinking, divergent thinking, insight, metaphors, and problem-solving).

\section{Exclusion Criteria}

Research studies were excluded if no exercise intervention was employed, self-report questionnaires of creative strengths and abilities were not accompanied by an observable laboratory measure of creative potential (McCutcheon, 1982), or if creativity was not the outcome variable (Hallihan \& Shu, 2011). Additionally, articles were excluded if the study population was comprised of nonhuman subjects.

\section{Search Strategy}

The following databases were searched between 1 January 2018 and 10 January 2018: PubMed, PsychInfo, and Google Scholar. MeSH keyword terms included exercise, physical exercise, physical activity, creativity, exercise and creativity, physical exercise and creativity, and physical activity and creativity. Subordinate terms included convergent thinking, divergent thinking, and insight problem-solving.

See Figure 1 for a flow diagram of the extracted studies from the computerized search. In total, 13 articles met the study criteria (Figure 1).
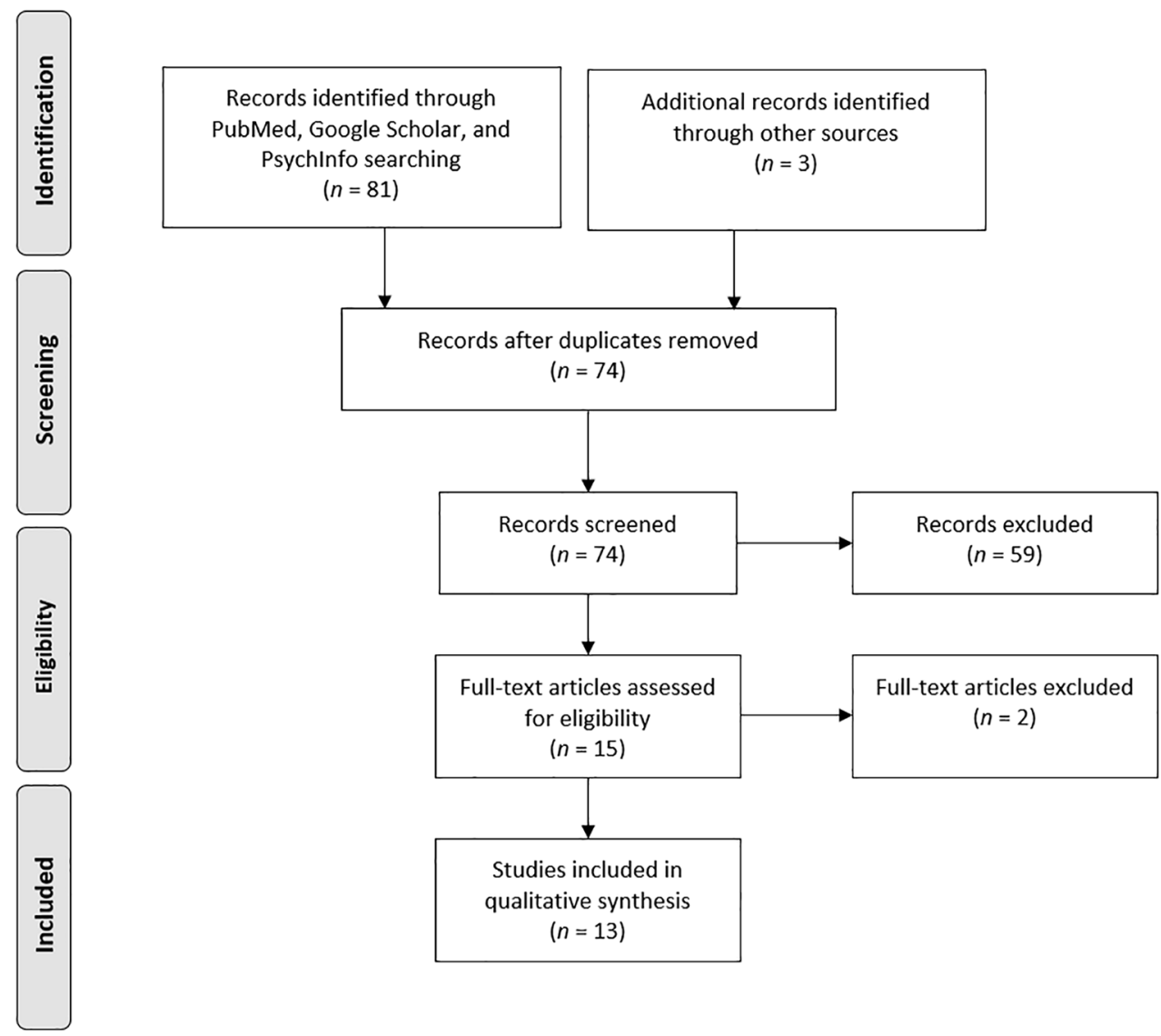

Figure 1. Flow diagram of the extracted studies. 


\section{Quality Evaluation}

Risk of bias/study quality was evaluated for each article using a checklist developed specifically for this study. The following checklist includes seven items with a yes (1) or no (0) response option and was constructed in accordance with the Cochrane Risk of Bias Tool (Higgins \& Green, 2008). This risk of bias checklist, as well as this entire systematic review, also adhered to the PRISMA checklist for reporting systematic reviews (except for Item 5, prospective registration of the systematic review). Two of the authors independently scored each study based on this checklist. Discrepancies were discussed until agreement was made. In situations when consensus did not occur, the two remaining authors were consulted, which facilitated consensus for all risk of bias items. The risk of bias items are as follows:

1. Was the physical activity manipulation controlled (e.g., completed in a laboratory setting, standardized by duration and intensity, and for interaction with other participants if administered in a group context)?

2. Was there evidence of reliability for the creativity measure(s) utilized?

3. Was there evidence of validity for the creativity measure(s) utilized?

4. Were creativity scoring and evaluation procedures robust to bias (e.g., blinded scoring completed by multiple researchers, provision of strong interrater reliability, and detailed or referenced?

5. Were random group assignment and/or counterbalancing procedures appropriate (e.g., were participants assigned to groups based on course enrollment, rather than random selection and were the order of creativity assessments randomized to ensure resistance to temporal artifacts or learning effects?) for the study design?

6. Did the intervention use a non-exercise control group or condition?

7. Were statistically appropriate/acceptable methods of data analysis used?

8. Were point estimates, standard deviations, confidence intervals, and/or effect sizes reported?

Items 2 and 3 required each manuscript to include an explicit description of evidence for reliability and validity of the creativity outcome assessments employed. The manuscript earned a 'no' ( 0 ) score for missing details regarding reliable/valid measures, which may have been utilized in some experiments, but were not adequately detailed per our quality evaluation criteria. A 'no' score for Item 7 was awarded to manuscripts that failed to use reasonable statistical methods for post-hoc analysis of outcomes. Statistically inappropriate decisions included reporting Pearson correlation coefficients for interrater reliability, failure to use the appropriate statistical tests and computing unpaired analyses of treatment effects on individual differences as a result of chronic training studies. Item 8 identified articles that neglected to report point estimates, confidence intervals, standard deviations, and/or effect sizes. The authors may have computed these values, but if all statistical results were not included in the publication, a 'no' score was given for Item 8.

The 13 included research studies were classified into categories based on cut points reflecting the degree of methodological bias considered for each individual study. Studies with a score of 6-8 (three studies) were classified as having low risk of bias. Studies with a score of 3-5 (eight studies) were classified as having moderate risk of bias. Studies with a score of $0-2$ (two studies) were classified as having a high risk of bias (Table 1). 
Table 1

Risk of Bias Assessment

\begin{tabular}{|c|c|c|c|c|c|c|c|c|c|}
\hline \multirow[b]{2}{*}{ Study } & \multicolumn{8}{|c|}{ Risk of Bias Item } & \multirow[b]{2}{*}{ Total } \\
\hline & 1 & 2 & 3 & 4 & 5 & 6 & 7 & 8 & \\
\hline Blanchette et al. (2005) & & $\mathrm{x}$ & $\mathrm{x}$ & $\mathrm{x}$ & $\mathrm{x}$ & $\mathrm{x}$ & & & 5 \\
\hline Colzato et al. (2013) & $x$ & $\mathrm{x}$ & $\mathrm{x}$ & $\mathrm{x}$ & $x$ & $x$ & & $x$ & 7 \\
\hline Curnow and Turner (1992) & $x$ & $\mathrm{x}$ & $\mathrm{x}$ & $x$ & & & $\mathrm{x}$ & & 5 \\
\hline Gondola and Tuckman (1985) [pilot study] & & $x$ & $\mathrm{x}$ & $\mathrm{x}$ & & & & & 3 \\
\hline Gondola (1986) & & $\mathrm{x}$ & $\mathrm{x}$ & & & & & & 2 \\
\hline Gondola (1987) & & $\mathrm{x}$ & $\mathrm{x}$ & & & & $\mathrm{x}$ & & 3 \\
\hline Herman-Tofler and Tuckman (1998) & & $\mathrm{x}$ & $\mathrm{x}$ & $\mathrm{x}$ & & & & $\mathrm{x}$ & 4 \\
\hline Hinkle et al. (1993) & & $x$ & $\mathrm{x}$ & $\mathrm{x}$ & $\mathrm{x}$ & & $\mathrm{x}$ & & 5 \\
\hline Oppezzo and Schwartz (2014) & $x$ & $\mathrm{x}$ & $\mathrm{x}$ & $x$ & $\mathrm{x}$ & $\mathrm{x}$ & $\mathrm{x}$ & $\mathrm{x}$ & 8 \\
\hline Ramocki (2002) & & & & & & $x$ & & & 1 \\
\hline Steinberg et al. (1997) & & $x$ & $\mathrm{x}$ & & & $\mathrm{x}$ & $\mathrm{x}$ & & 4 \\
\hline Tuckman and Hinkle (1986) & & $x$ & $x$ & & & & $\mathrm{x}$ & & 3 \\
\hline Zhou et al. (2017) & $\mathrm{x}$ & & & $\mathrm{x}$ & $\mathrm{x}$ & $\mathrm{x}$ & $\mathrm{x}$ & $\mathrm{x}$ & 6 \\
\hline
\end{tabular}

Note. Item 1: Was the physical activity manipulation controlled (e.g., completed in a laboratory setting, standardized by duration and intensity, and for interaction with other participants if administered in a group context)? Item 2: Was there evidence of reliability for the creativity measure(s) utilized? Item 3: Was there evidence of validity for the creativity measure(s) utilized? Item 4: Were creativity scoring and evaluation procedures robust to bias (e.g., blinded scoring completed by multiple researchers, provision of strong interrater reliability, and detailed or referenced? Item 5: Were random group assignment and/or counterbalancing procedures appropriate (e.g., were participants assigned to groups based on course enrollment, rather than random selection and were the order of creativity assessments randomized to ensure resistance to temporal artifacts or learning effects?) for the study design? Item 6: Did the intervention use a non-exercise control group or condition? Item 7: Were statistically appropriate/acceptable methods of data analysis used? Item 8: Were point estimates, standard deviations, confidence intervals, and/or effect sizes reported?

\section{Data Extraction}

A data extraction table for the included research studies was created to provide a brief description of author names and publication date, sample characteristics, research design, creativity measures used and length of creativity assessment period, relevant creativity parameters assessed, exercise modality, intensity and duration, methods used for scoring creative products, as well as study outcomes and conclusions (Table 2). 


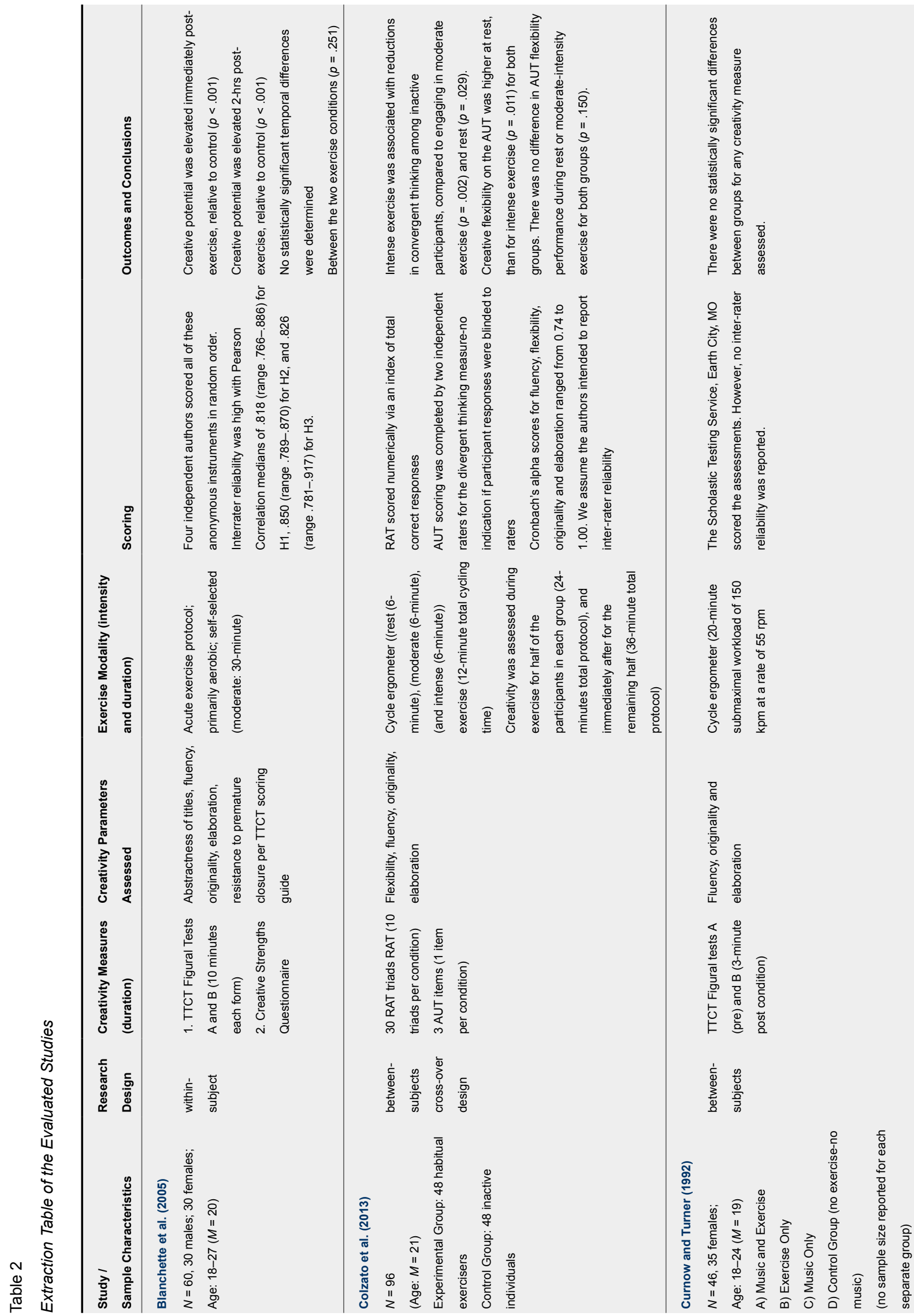




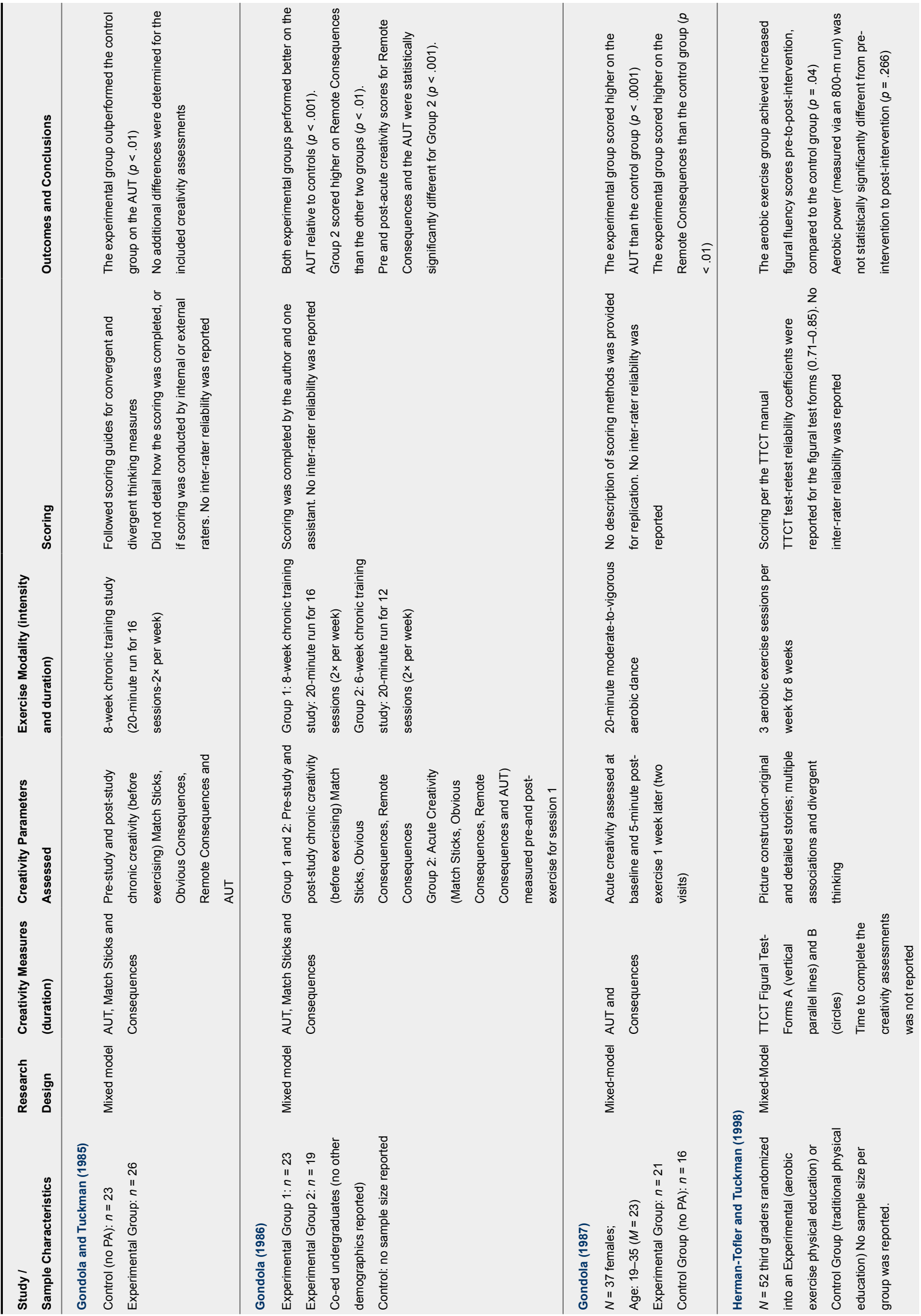




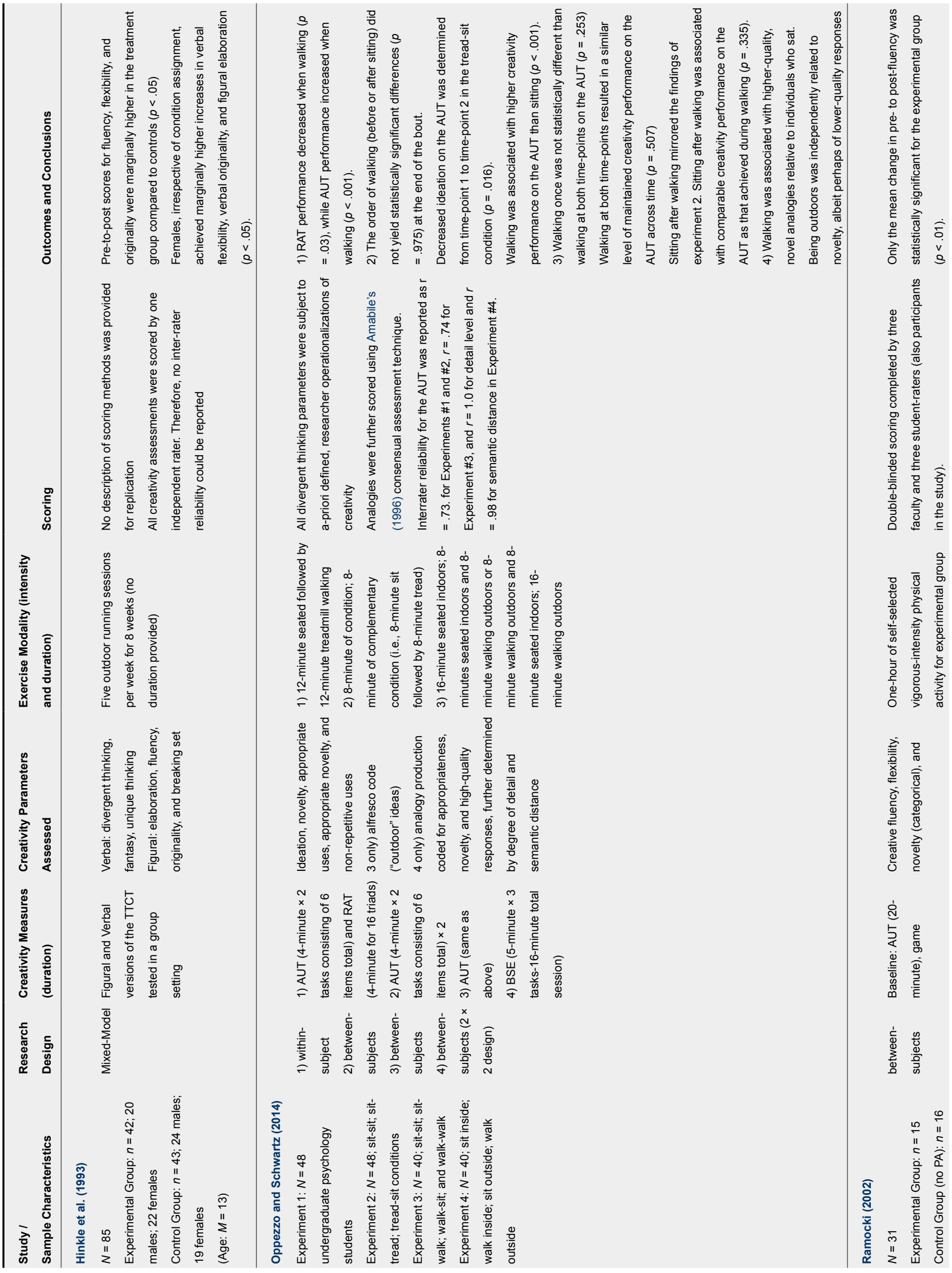




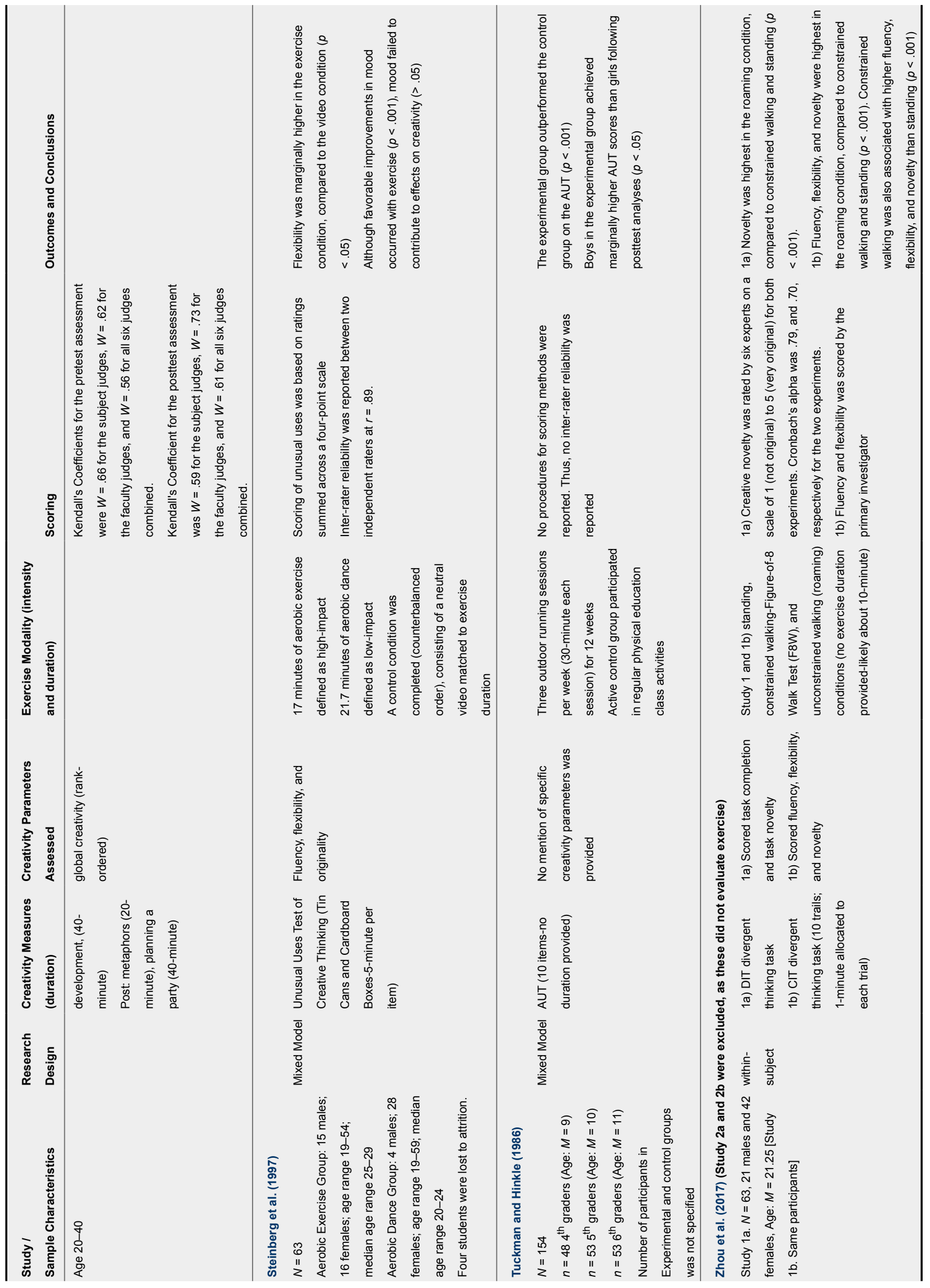




\section{Results}

\section{Creativity Assessments}

There are many different creativity assessments which may be utilized to experimentally assess acute creative potential in the laboratory. Four studies employed the Torrance Tests of Creative Thinking (TTCT) Figural Tests A and B (Torrance, Ball, \& Safter, 1966), with one utilizing both the Figural and Verbal forms. Eight studies assessed divergent thinking, and two studies employed both divergent and convergent thinking assessments. Two studies evaluated analogy generation or production of metaphors. Assuming that these measures all demonstrate comparable quality in assessing certain aspects of creativity, caution should be observed when interpreting these results for practical generalizability.

\section{Study Description}

Among the 13 manuscripts selected for this systematic review, all evaluated a hypothesized relationship between exercise manipulation and creativity performance. Of the 13 articles, three were published after 2013, two were published from 2002 to 2005, and eight were published from 1985 to 1998. Nine studies evaluated exercise and creativity within college-aged individuals, three studies assessed elementary and/or middle school children, and one study utilized a sample of adults at least 18 years of age. Nearly one-third of the included studies failed to report sample sizes per experimental or control group assignment. To this end, due to the substantial heterogeneity across study quality and methodology, a meta-analytic approach was not appropriate to include, and a qualitative review of research studies was chosen to avoid further convolution of conclusions suggested in the existing research on exercise and creativity (Walker, Hernandez, \& Kattan, 2008). Three studies utilized a traditional within-subject design, four used a between-subjects protocol, one used a two-visit between-subjects design (Gondola, 1987), and four mixed-method studies implemented aerobic running sessions lasting 6-12 weeks, consisting of both between-and within-subject comparisons. One study was initially designed to employ a between-subjects design, but then collapsed the two experimental groups at the conclusion of the study to accrue a more robust sample size for analysis of treatment effects (Steinberg et al., 1997).

\section{Risk of Bias}

Among the 13 experimental studies, 23\% $(n=3 / 13)$ were determined to contain low risk of bias, $62 \%(n=8 / 13)$ of the included studies were considered to be of moderate risk of bias, and $15 \%(n=2 / 13)$ were considered to have been conducted with high risk of bias.

\section{Main Outcome Results Across Exercise Intensities}

Among the 13 evaluated studies in this systematic review, 12 demonstrated some evidence of a beneficial effect of exercise on creativity. Further details on select studies, along with their limitations, are noted in the Discussion section. Among the 13 studies, eight evaluated moderate intensity and eight evaluated vigorous intensity exercise (two studies evaluated both moderate and vigorous intensity exercise). Regarding the eight studies focused on moderate intensity exercise, three demonstrated a significant effect of exercise on divergent thinking, specifically immediate and delayed improvements in figural creativity (Blanchette et al., 2005), increased fluency and novelty (defined as original and contextually appropriate) during and following exercise compared to rest, increases in high-quality analogies during exercise, an increase in novelty when walking out- 
doors (Oppezzo \& Schwartz, 2014), as well as when roaming (free ambulation), or walking unconstrained. Notably, constrained walking was also shown to increase novelty compared to rest, but novel responses while walking along a predetermined path were statistically significantly lower than roaming unconstrained. A moderate intensity cycling protocol was shown to have no statistically significant influence on figural creativity measured by fluency, originality, and elaboration (although Curnow and Turner (1992) suggested weak support for a fluency effect at "the .05 level"). Among the seven studies employing vigorous exercise protocols, six demonstrated a significant effect. Convergent and divergent thinking was elevated among female dancers (although no scoring information was provided; Gondola, 1987). Alternatively, convergent thinking performance was reduced among inactive participants during intense cycling exercise, compared to both moderate intensity and rest conditions. Rest and moderate intensity cycling did not produce statistically significant outcomes in convergent thinking. Additionally, divergent thinking was higher during rest than intense exercise for inactive and active participants, with no statistically significant difference found between moderate cycling and rest (Colzato, Szapora, Pannekoek, \& Hommel, 2013). Ramocki (2002) demonstrated statistical significance for divergent thinking fluency improvements following vigorous intensity exercise. This study was vulnerable to substantial bias, receiving a score of "1" on our quality assessment, so these findings should be cautiously interpreted. Steinberg et al. (1997) indicated divergent thinking flexibility was increased among participants; however, individuals completed either "low-impact, rhythmic stretching," or "high-impact" aerobic dance, and were analyzed as a homogenous experimental group following the conclusion of the study. Therefore, those findings cannot be considered meaningful.

High intensity, chronic training studies (aerobic running durations of 20-30 minutes with 2-5 sessions per week) were shown to enhance divergent thinking performance (Gondola, 1986; Gondola \& Tuckman, 1985; Herman-Tofler \& Tuckman, 1998; Hinkle et al., 1993; Tuckman \& Hinkle, 1986), with studies evaluating children demonstrating marginal gender-specific differences, specifically positing that girls may be more responsive to training-induced improvements in figural elaboration (Hinkle et al., 1993) and verbal flexibility and originality, with boys perhaps more likely to outperform girls on general divergent thinking measures (Tuckman \& Hinkle, 1986).

\section{Discussion}

\section{Results on Exercise and Creativity}

Steinberg et al. (1997) evaluated aerobic dance and stretching lasting approximately 17-20 minutes in duration. The exercise protocol consisted of 6 minutes warm-up, 6 minutes of an aerobics class and 6 minutes of cool-down exercises. The aerobic exercise procedure was classified as "high impact," while the rhythmic dancing was considered low-impact and consisted of a 4-5-minute warm-up, a 14-minute dance period, and 3-4 minutes of cooling down. Despite the decision to administer two distinct exercise protocols, the aerobics group and dancing group were combined into an aggregate sample following experimentation. This post-hoc deviation from the initial study design is problematic, as diverse modalities and intensities may uniquely influence the influence of physical exercise on creative cognitions. Creativity was also only measured post-exercise for this experiment, as further counterbalancing of creativity tasks would have rendered the procedure "too complicated and time-consuming." 
Other exercise protocols permitted participants to engage in weight lifting, as well as any accessible form of aerobic indoor or outdoor exercise (e.g., swimming, running, brisk walking, cycling, or stair climbing), continuous or intermittent, with a troubling dismissal of experimental control. Even among studies with adequate experimental control, the exercise regimen remained flawed, as one study asked participants to engage in both moderate and intense exercise within the same bout, failing to indicate that measurements of creative potential may be distorted by residual fatigue, particularly for inactive individuals randomized to complete the intense condition prior to the moderate intensity condition.

A crucial point must be considered as researchers aim to extend the field creativity and exercise. Specifically, the time-point at which creativity is assessed relative to the exercise bout warrants scrupulous empirical attention. If exercise is expected to exert evaluable effects on creative potential, then experiments must be designed to illuminate how, why, when, and for whom these effects may occur. Researchers often assess creativity before and after a single exercise bout (Curnow \& Turner, 1992; Gondola, 1987; Ramocki, 2002) or multi-visit training program (Gondola, 1986; Gondola \& Tuckman, 1985; Herman-Tofler \& Tuckman, 1998; Hinkle et al., 1993). Although, some authors report testing creativity following the exercise bout, with no baseline assessment (Blanchette et al., 2005; Steinberg et al., 1997), while others administer a concurrent task protocol (Oppezzo \& Schwartz, 2014; Zhou et al., 2017), to identify the relationship between creative cognitions activated during the transient stimulation of exercise. Research has also investigated timing differences between creativity assessed before and after, or during and immediately following acute exercise of moderate versus highintensity (Colzato, Szapora, Pannekoek, \& Hommel, 2013). There is utility in assessing potential temporal relationships across numerous research projects, or perhaps in a single research endeavor. To that end, it is troubling that no study, to date, has attempted to assess creativity before, during, immediately after, and many hours following exercise to test individual responses in a multi-visit study, in addition to isolating between-group differences contingent upon the timing of exercise.

\section{Informed Methods of Inquiry}

All articles included reported that creativity was either augmented or decreased as a function of exercise manipulation, failing to underscore the reservations inherent in creativity tasks designed to evaluate specific creative correlates (i.e., divergent thinking, convergent thinking, insight, imagination, analogy, metaphor, etc.). Therefore, stating that creativity, in general, is improved or impaired by exercise, is as problematic as concluding that exercise improves or impairs a measure as intricate as intelligence, for science must always aim to specify precisely which outcome characteristics are changing in line with research interventions.

Three studies matched the duration of the creativity task with the duration of exercise. These studies employed between-subject designs, but the practice of time-matching creativity assessments to exercise stimulus in between-subject designs is a compelling direction for researchers to consider, especially when evaluating exercise-induced cognitive resource depletion, and/or residual effects of exercise persisting for a shorter creativity assessment, with a creativity measure requiring sustained mental resource allocation equivalent in length to the exercise bout.

Scoring of creativity tasks was inadequate for the majority of included manuscripts. Perhaps authors adhered to best research practices, such as use of a validated scoring manual, blinded rating, utilization of more than one rater, or using more objective statistical measures to denote the originality facet of creativity, such as identifying 
cut-point percentages, using the top-three method, or calculating a creativity quotient (Plucker, Qian, \& Schmalensee, 2014; Plucker, Qian, \& Wang, 2011; Silvia, Martin, \& Nusbaum, 2009; Snyder, Mitchell, Bossomaier, \& Pallier, 2004). It is crucial that participants be prohibited from scoring sample responses, even if these individuals are not scoring their own responses and are blinded to the identities of the other participants. One research study utilized this approach, following a creativity assessment protocol, administered in a group format, making rendering exclusive anonymity impossible. Other studies neglected to describe the scoring protocol, or the selection criteria and verification procedures used to identify expert scorers, which effectively obscures paths for subsequent research to follow in replication and refinement of methodological decisions.

\section{Limitations}

Limitations of this review include the collaborative efforts of only two researchers to search databases and access relevant manuscripts. Searching three databases is another potential weakness, as it is possible research experiments fitting our inclusionary criteria may have been overlooked. However, we feel confident the search strategies employed were sufficiently comprehensive. Moreover, the full text of the exercise and creativity experiments extracted were read in full, and reference lists were crossed-checked by each of the primary researchers to ensure a parsimonious, yet extensive review of the literature was satisfied. Although quality assessment methods were developed in alignment with the PRISMA checklist for reporting systematic reviews, it is possible some items were overlooked which may have increased or reduced the bias scores for these studies. Further, the items developed to indicate risk of bias were formulated by the two researchers involved in this review. Additional researchers may have provided supplementary insight to refine the items to reflect higher quality evaluation methods than those achieved herein. Nevertheless, we feel the present evaluations are contextually appropriate, fair, and may engender continued discussion and more informed experimental practices.

\section{Strengths of Exercise and Creativity Research}

Extant empirical investigations of the plausible relationship between exercise and creativity have provided a robust platform for continued exploration. Thus far, the field is beginning to depart from a general recognition of conjectural anecdotes suggesting physical movement may liberate mental constraints and encourage creative cognitions and is approaching an evidenced-based understanding. The articles included herein provide modest support for exercise to meaningfully impact creative thinking. Beyond the inclusion of various exercise modalities, durations, and environments (detailed in the methods section) by which to assess creativity, there are specific recommendations from well-conducted research that should inform future methods of inquiry in this arena.

Oppezzo and Schwartz (2014) theorized that an observed interplay between comfortable, self-selected ambulatory exercise and creativity may be associated with improvements in positive mood related to the mind-freeing nature of exercise and physical movement, which may also play a role in the activation of associative memory processes conducive to originality at the expense of conventional, ideation. Moreover, the authors additionally suggest that convergent thinking may require a higher degree of cognitive control, which would explain decrements in convergent thinking during acute exercise (Oppezzo \& Schwartz, 2014). Relatedly, Colzato et al. (2013), examined the effects of exercise temporality and intensity on divergent and convergent thinking assessed both during and following acute exercise, demonstrating that sedentary individuals achieved higher convergent thinking during and after both moderate-intensity exercise and rest conditions compared to high-intensity exercise, and that habitual exercisers achieved higher flexibility in divergent thinking during rest compared to 
intense exercise, proposing that complex cognitions associated with convergent thinking and flexibly overcoming mental fixation may be vulnerable to depletion of cognitive control resources (Colzato, 2013).

\section{Recommendations for Future Research}

Research has yet to definitively uncover why and how exercise may influence the global construct of cognitive creativity. However, the plausibility for exercise to exert measurable effects is encouraging, as facilitative mindbody connections have been extensively proposed as mechanisms for improvements in memory (Frith, Sng, \& Loprinzi, 2017; Sng, Frith, \& Loprinzi, 2017) and cognition (Chang, Labban, Gapin, \& Etnier, 2012; Etnier et al., 1997; Loprinzi \& Kane, 2015). In addition, much anecdotal evidence alludes to an influential relationship between physical exercise and cognitive creativity. Moreover, the underpinnings of movement and mental resource allocation are suggested to activate shared neural pathways, which further highlights the dynamic complexity of human physiology and cognition. Despite considerable efforts to illuminate this association, the results remain inconclusive. Future research should extend scientific understanding of such neural mechanisms in the context of exercise and creativity (Gonen-Yaacovi et al., 2013). Functional imaging of prefrontal areas of the human brain at rest, during a creativity assessment following a rest period, and during a creativity following exercise may facilitate a deeper understanding of neural activation subserving creative cognition. Additionally, baseline individual differences in complex-cognitions related to creativity (such as inhibition and flexibility), which may be captured using executive functioning assessments (Gilbert \& Burgess, 2008), should be measured to determine whether baseline cognition mediates relationships observed between exercise and creativity thinking.

There appears to be weak to modest support for acute, moderate-intensity exercise to benefit creativity. Highintensity exercise appears to induce a detrimental effect on convergent creativity when the creativity task and vigorous exercise are administered concurrently in unfit individuals. Interestingly, rest, or the absence of exercise may have a similar deleterious influence on convergent creativity among regular exercisers, however subsequent research should attempt to further question these speculations by examining valence-related effects of exercising on creativity scores. Specifically, when habituated, and perhaps enjoyable behaviors (e.g., exercise) are withheld, is substitution of a less enjoyable activity (e.g., forced inactivity) in an environment conducive to exercise, capable of inducing negative affect or amotivation, which may act synergistically to reduce creative performance? Conversely, moderate-intensity exercise has also been shown to impair convergent thinking performance, suggesting that, perhaps, convergent tests of creativity are susceptible to exercise-driven depletion of mental resources necessary to complete the task, or reductions in attention, motivation, or affectual responses. Although, it is possible these speculations are entirely misguided, as one study found exercise may be capable of enhancing creativity, independently of changes in mood state. Nevertheless, results from the studies included herein tend to suggest a potential immediate and residual effect of exercise participation on creative performance, specifically divergent fluency and flexibility assessed in the laboratory, with improvements in divergent flexibility more equivocal. Oppezzo and Schwartz (2014) provided a practical interpretation of their findings, proposing that although walking may make people more talkative, fluency alone cannot equate to creativity. Therefore, the authors computed an additional analysis suggesting that appropriate novelty was elevated within individuals who walked, not only because these individuals were more fluent, but because their total ideation volume contained more divergent responses. To this end, it is prudent to consider the totality of the existing exercise and creativity research, fraught with shortcomings, but also promising trajectories for continued, careful investigation. 
Aerobic training studies lasting at least 6 weeks in duration, and with at least two exercise session per week may have some utility on influencing adult and childhood creativity, however, these findings should be interpreted with caution, as many studies failed to employ a non-exercise control arm, or even standardize the exercise protocol within the experimental group. Additionally, one 8-week study showed no statistically significant improvements in 800-meter run performance, suggesting figural fluency was marginally augmented in the absence of fitness improvements. To date, it is unclear whether exercise benefits, undermines, or has no bearing on creative functioning. Therefore, research studies should focus on first identifying relationships in controlled laboratory environments, more robust to confounding factors unaccounted for in outdoor environments. Further, while it was unclear if creativity assessments were always administered in either an individualized or group setting. For all training studies, the exercise portion was completed in a group format, which may exert unintended effects on motivation, affect, and effort. All training studies included in this review failed to report one or all of the following statistical indices of practically meaningful results, including effect sizes, confidence intervals, or point estimates. Reliance on p-values is insufficient, incomplete, and misleading for any research agenda (Wilkinson, 2014). Moreover, none of the four training studies reviewed were conducted in a laboratory setting, which would be less of a limitation if compliance to the training protocol was detailed, or perhaps, if evidence for habitual exercise to benefit creative thinking was well established in the literature. Again, the vast majority of conclusions presented within exercise and creativity research deteriorate in plausibility as fragile study designs and analytic decisions are applied, perhaps for the purposes serving feasibility, but undeniably at the cost of scientific progress.

Despite the enigmatic challenges that emerge when assessing creativity in acute, laboratory settings, controlled measurement of creative potential is imperative for researchers to accumulate a comprehensive understanding of the various manipulations designed to address proposed associations between exercise and creativity. Controlled, empirical work will allow researchers to provide compelling evidence for theoretical mechanisms underlying the proposed exercise-creativity link. We suggest that an exercise-driven approach to measuring creativity is an exciting avenue for continued scientific investigation of the longitudinal effects of exercise on the creative person, including motivation and personality factors (Cropley, 2000), as well as acute and chronic effects of exercise on creativity performance across various age groups.

Future research is warranted to assess the influence of physical activity in early childhood on movement-based creativity outcomes, such as Torrance's Thinking Creatively in Action and Movement Test (TCAM), which was designed on the precedent that young children manipulate and organize their thoughts in expressive, kinesthetic actions, as their proficiency in verbalization, writing, or drawing may be less cultivated at the preschool age (Torrance, 1981). Regarding the experiments reviewed herein, three provided evidence for chronic physical exercise to confer higher figural and verbal creativity (measured via the TTCT and AUT) in children between the third and sixth grade (Herman-Tofler \& Tuckman, 1998; Hinkle \& Sampson, 1993; Tuckman \& Hinkle, 1986). To our knowledge, no acute exercise studies have been conducted in childhood populations. Further, exercise and creativity research extended to younger populations is warranted. Notably, however, young children may not provide responses that accurately reflect their creative capacity in words or drawings, but, rather, are perhaps more likely to act out their thoughts using symbolic, representational movement (Zachopoulou, Makri, \& Pollatou, 2009). Torrance proposed that bodily motion is an indelibly potent strategy for unveiling creativity in early childhood populations (Torrance, 1981). Thus, it is worthwhile for researchers to consider that physical activity may promote creative, associative thinking, particularly in physical domains, perhaps representing an interface between tactile, sensorimotor representations of developing complex cognitions. 
Research investigating the utility for physical activity to influence creative cognitions in older adulthood is also warranted. Fifty-nine years is the oldest age reported to have been assessed in the exercise and creativity experiments reviewed herein, with the age range of that experiment ranging from 19 to 59 (Steinberg et al., 1997). Future work focusing specifically on potential exercise-induced influences on creativity in aging populations is important. Previous search suggests that aging individuals with dementia generally exhibit impaired performance on laboratory assessments of divergent thinking (Cruz de Souza et al., 2010; Hart \& Wade, 2006). Perhaps regular exercise may attenuate dementia symptomology and aging-induced frontal-lobe dependent decrements in cognition (Colcombe \& Kramer, 2003), as the prefrontal cortex shares functional neural connections with motor regions in the brain (Cole et al., 2013), which may facilitate the maintenance of higher-order mental functions, including creativity, in later life.

\section{Conclusions}

Weak evidence exists, to date, in support of the proposed relationships between exercise and creative thinking processes. Inferences of causality are difficult to accept, given the paucity of well-designed experiments in this domain of scientific investigation. Exercise and creativity researchers should first align their methodologies with unbiased measurement and evaluation practices, carefully designed to answer prudent explanatory questions. Restructuring the current framework requires a swift dismissal of ideological barriers to discovery, namely the conflation of creativity with divergent thinking, as well as unmitigated advancement into the dense tangle of speculative discourse aiming to contrive tenuous links between creativity and exercise. Experiments continue to employ minimal standardization, laboratory control, resistance to confounding, and rigorous, detailed scoring procedures, leaving the same questions unanswered and limiting valid conclusions. The prospects for growth and development in research examining creativity and exercise associations are astronomical, but only if the field commits to consistency and quality when assessing the potential for such relationships.

\section{Funding}

The authors have no funding to report.

\section{Competing Interests}

The authors have declared that no competing interests exist.

\section{Acknowledgments}

We declare no conflicts of interest and no funding was used to prepare this manuscript.

\section{References}

Amabile, T. M. (1996). Creativity in context: Update to "The social psychology of creativity". Boulder, CO, USA: Westview Press.

Berkowitz, G. (2014). Convergent and divergent thinking. In Encyclopedia of special education. 
Blanchette, D. M., Ramocki, S. P., O'del, J. N., \& Casey, M. S. (2005). Aerobic exercise and creative potential: Immediate and residual effects. Creativity Research Journal, 17, 257-264. https://doi.org/10.1207/s15326934crj1702\&3_10

Chang, Y. K., Labban, J. D., Gapin, J. I., \& Etnier, J. L. (2012). The effects of acute exercise on cognitive performance: A meta-analysis. Brain Research, 1453, 87-101. https://doi.org/10.1016/j.brainres.2012.02.068

Colcombe, S., \& Kramer, A. F. (2003). Fitness effects on the cognitive function of older adults: A meta-analytic study. Psychological Science, 14(2), 125-130. https://doi.org/10.1111/1467-9280.t01-1-01430

Cole, M. W., Reynolds, J. R., Power, J. D., Repovs, G., Anticevic, A., \& Braver, T. S. (2013). Multi-task connectivity reveals flexible hubs for adaptive task control. Nature Neuroscience 16(9), 1348-1355. https://doi.org/10.1038/nn.3470

Colzato, L. S., Szapora, A., Pannekoek, J. N., \& Hommel, B. (2013). The impact of physical exercise on convergent and divergent thinking. Frontiers in Human Neuroscience, 7, Article 824. https://doi.org/10.3389/fnhum.2013.00824

Cropley, A. J. (2000). Defining and measuring creativity: Are creativity tests worth using? Roeper Review, 23(2), 72-79. https://doi.org/10.1080/02783190009554069

Cruz de Souza, V. E., Bertoux, M., Czernecki, V., Funkiewiez, A., Allali, G., . . Leroy, B., et al. (2010). Poor creativity in frontotemporal dementia: A window into the neural bases of the creative mind. Neuropsychologia, 48, 3733-3742. https://doi.org/10.1016/j.neuropsychologia.2010.09.010

Csikszentmihalyi, M. (1999). 16 implications of a systems perspective for the study of creativity. In R. J. Sternberg (Ed.) Handbook of Creativity, 313-335. Cambridge, United Kingdom: Cambridge University Press.

Curnow, K. E., \& Turner, E. T. (1992). The effect of exercise and music on the creativity of college The Journal of Creative Behavior, 26, 50-52. https://doi.org/10.1002/j.2162-6057.1992.tb01156.x

Dietrich, A., \& Kanso, R. (2010). A review of EEG, ERP, and neuroimaging studies of creativity and insight. Psychological Bulletin, 136(5), 822-848. https://doi.org/10.1037/a0019749

Etnier, J. L., Salazar, W., Landers, D. M., Petruzzello, S. J., Han, M., \& Nowell, P. (1997). The influence of physical fitness and exercise upon cognitive functioning: A meta-analysis. Journal of sport and Exercise Psychology, 19, $249-277$. https://doi.org/10.1123/jsep.19.3.249

Frith, E., Sng, E., \& Loprinzi, P. D. (2017). Randomized controlled trial evaluating the temporal effects of high-intensity exercise on learning, short-term and long-term memory, and prospective memory. European Journal of Neuroscience, 46(10), 2557-2564. https://doi.org/10.1111/ejn.13719

Gilbert, S. J., \& Burgess, P. W. (2008). Executive function. Current Biology, 18(3), R110-R114. https://doi.org/10.1016/j.cub.2007.12.014

Gondola, J. C. (1986). The enhancement of creativity through long and short term exercise. Journal of Social Behavior \& Personality, 1, 77-82.

Gondola, J. C. (1987). The effects of a single bout of aerobic dancing on selected tests of creativity. Journal of Social Behavior \& Personality, 2, 275-278.

Gondola, J. C., \& Tuckman, B. W. (1985). Effects of a systematic program of exercise on selected measures of creativity. Perceptual and Motor Skills, 60(1), 53-54. https://doi.org/10.2466/pms.1985.60.1.53 
Gonen-Yaacovi, G., De Souza, L. C., Levy, R., Urbanski, M., Josse, G., \& Volle, E. (2013). Rostral and caudal prefrontal contribution to creativity: A meta-analysis of functional imaging data. Frontiers in Human Neuroscience, 7, Article 465. https://doi.org/10.3389/fnhum.2013.00465

Guilford, J. P. (1950). Creativity. American Psychologist, 5(9), 444-454. https://doi.org/10.1037/h0063487

Hallihan, G. M., \& Shu, L. H. (2011, January). Creativity and long-term potentiation: Implications for design. Proceedings of the ASME 2011 International Design Engineering Technical Conferences \& Computers and Information in Engineering Conference, Washington D.C., USA, pp. 1-12.

Hart, R. P., \& Wade, J. (2006). Divergent thinking in Alzheimer's and frontotemporal dementia. Neuropsychology, Development, and Cognition. Section B, Aging, Neuropsychology and Cognition, 13, 281-290. https://doi.org/10.1080/13825580490904246

Herman-Tofler, L. R., \& Tuckman, B. W. (1998). The effects of aerobic training on children's creativity, self-perception, and aerobic power. Child and Adolescent Psychiatric Clinics of North America, 7(4), 773-790. Article viiihttps://doi.org/10.1016/S1056-4993(18)30211-6

Higgins, J. P. T., \& Green, S. M. (2008). Cochrane handbook for systematic reviews of interventions. http://www.cochrane-handbook.org.

Hinkle, J. S., Tuckman, B. W., \& Sampson, J. P. (1993). The psychology, physiology, and creativity of middle school aerobic exercisers. Elementary School Guidance \& Counseling, 28(2), 133-145.

Loprinzi, P. D., \& Kane, C. J. (2015). Exercise and cognitive function: A randomized controlled trial examining acute exercise and free-living physical activity and sedentary effects. Mayo Clinic Proceedings, 90(4), 450-460. https://doi.org/10.1016/j.mayocp.2014.12.023

McCutcheon, L. E. (1982). Does running make people more creative? Journal of Sport Behavior, 5, $202-206$.

Mumford, M. D. (2003). Where have we been, where are we going? Taking stock in creativity research. Creativity Research Journal, 15, 107-120. https://doi.org/10.1207/S15326934CRJ152\&3_01

Murad, M. H., Montori, V. M., loannidis, J. P., Jaeschke, R, ., Devereaux, P. J., . . Prasad, K.et al. (2014). How to read a systematic review and meta-analysis and apply the results to patient care: Users' guides to the medical literature. JAMA, 312(2), 171-179. https://doi.org/10.1001/jama.2014.5559

Oppezzo, M., \& Schwartz, D. L. (2014). Give your ideas some legs: The positive effect of walking on creative thinking. The Journal of Experimental Psychology: Learning, Memory, and Cognition, 40(4), 1142-1152. https://doi.org/10.1037/a0036577

Plucker, J. A., Qian, M., \& Schmalensee, S. L. (2014). Is what you see what you really get? Comparison of scoring techniques in the assessment of real-world divergent thinking. Creativity Research Journal, 26, 135-143. https://doi.org/10.1080/10400419.2014.901023

Plucker, J. A., Qian, M., \& Wang, S. (2011). Is originality in the eye of the beholder? Comparison of scoring techniques in the assessment of divergent thinking. Journal of Creative Behavior, 45, 1-22.

https://doi.org/10.1002/j.2162-6057.2011.tb01081.x 
Ramocki, S. P. (2002). Creativity interacts with fitness and exercise. Physical Educator, 59, 8.

Runco, M. A. (2008). Commentary: Divergent thinking is not synonymous with creativity. Psychology of Aesthetics, Creativity, and the Arts, 2, 93-96. https://doi.org/10.1037/1931-3896.2.2.93

Silvia, P. J., Martin, C., \& Nusbaum, E. C. (2009). A snapshot of creativity: Evaluating a quick and simple method for assessing divergent thinking. Thinking Skills and Creativity, 4, 79-85. https://doi.org/10.1016/j.tsc.2009.06.005

Sng, E., Frith, E., \& Loprinzi, P. D. (2017). Temporal effects of acute walking exercise on learning and memory function. The American Journal of Health Promotion, 32(7), 1518-1525. https://doi.org/10.1177/0890117117749476

Snyder, A., Mitchell, J., Bossomaier, T., \& Pallier, G. (2004). The creativity quotient: An objective scoring of ideational fluency. Creativity Research Journal, 16, 415-419. https://doi.org/10.1080/10400410409534552

Stein, M. I. (1953). Creativity and culture. The Journal of Psychology, 36(2), 311-322. https://doi.org/10.1080/00223980.1953.9712897

Steinberg, H., Sykes, E. A., Moss, T., Lowery, S., LeBoutillier, N., \& Dewey, A. (1997). Exercise enhances creativity independently of mood. British Journal of Sports Medicine, 31(3), 240-245. https://doi.org/10.1136/bjsm.31.3.240

Torrance, E. P. (1981). Thinking creatively in action and movement. Bensenville, IL, USA: Scholastic Testing Service.

Torrance, E. P., Ball, O. E., \& Safter, H. T. (1966). Torrance tests of creative thinking. Princeton, NJ, USA: Personnel Press.

Tuckman, B. W., \& Hinkle, J. S. (1986). An experimental study of the physical and psychological effects of aerobic exercise on schoolchildren. Health Psychology, 5(3), 197-207. https://doi.org/10.1037/0278-6133.5.3.197

Walker, E., Hernandez, A. V., \& Kattan, M. W. (2008). Meta-analysis: Its strengths and limitations. The Cleveland Clinic Journal of Medicine, 75(6), 431-439. https://doi.org/10.3949/ccjm.75.6.431

Wilkinson, M. (2014). Distinguishing between statistical significance and practical/clinical meaningfulness using statistical inference. Sports Medicine, 44(3), 295-301. https://doi.org/10.1007/s40279-013-0125-y

Zachopoulou, E., Makri, A., \& Pollatou, E. (2009). Evaluation of children's creativity: Psychometric properties of Torrance's' Thinking Creatively in Action and Movement Test. Early Child Development and Care, 179(3), 317-328. https://doi.org/10.1080/03004430601078669

Zhou, Y., Zhang, Y., Hommel, B., \& Zhang, H. (2017). The impact of bodily states on divergent thinking: Evidence for a control-depletion account. Frontiers in Psychology, 8, Article 1546. https://doi.org/10.3389/fpsyg.2017.01546

\section{About the Authors}

Emily Frith, PhD, is a recent graduate of the doctoral program in the Department of Health, Exercise Science and Recreation Management at the University of Mississippi.

Seungho Ryu, M.S., is a PhD student in the Department of Health, Exercise Science and Recreation Management at the University of Mississippi. 
Minsoo Kang, PhD, is Professor in the Department of Health, Exercise Science and Recreation Management at the University of Mississippi.

Paul Loprinzi, PhD, is an associate professor in the Department of Health, Exercise Science and Recreation Management at the University of Mississippi. He is the director of the Exercise \& Memory Laboratory. 